\title{
In vitro method for prediction of plaque reduction by dentifrice
}

\author{
Bruce Tepper ${ }^{\mathrm{a}, *, 1}$, Brian Howard ${ }^{\mathrm{b}, *}$, Daniel Schnell ${ }^{\mathrm{c}}$, Lisa Mills ${ }^{\mathrm{a}}$, Jian $\mathrm{Xu}^{\mathrm{d}}$ \\ a Microbiology Capability Organization, The Procter E Gamble Company, 8700 Mason-Montgomery Road, Mason, OH 45040, USA \\ b Life Sciences Innovation, The Procter \& Gamble Company, 8700 Mason-Montgomery Road, Mason, OH 45040, USA \\ c Statistics and Data Management, The Procter E Gamble Company, 8700 Mason-Montgomery Road, Mason, OH 45040, USA

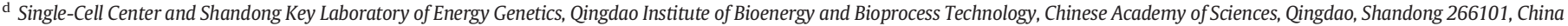

\section{A R T I C L E I N F O}

\section{Article history:}

Received 14 May 2015

Received in revised form 26 June 2015

Accepted 27 June 2015

Available online 4 July 2015

\section{Keywords:}

Oral biofilm

Biofilm cleaning

454 pyrosequencing

\begin{abstract}
A B S T R A C T
An in vitro Particle Based Biofilm (PBB) model was developed to enable high throughput screening tests to predict clinical plaque reduction. Multi-species oral biofilms were cultured from pooled stimulated human saliva on continuously-colliding hydroxyapatite particles. After three days PBBs were saline washed prior to use in screening tests. Testing involved dosing PBBs for 1 min followed by neutralization of test materials and rinsing. PBBs were then assayed for intact biofilm activity measured as ATP. The ranking of commercial dentifrices from most to least reduction of intact biofilm activity was Crest ProHealth Clinical Gum Protection, Crest ProHealth, Colgate Total and Crest Cavity Protection. We demonstrated five advantages of the PBB model: 1 ) the ATP metric had a linear response over $\geq 1000$-fold dynamic range, 2 ) potential interference with the ATP assay by treatments was easily eliminated by rinsing PBBs with saline, 3 ) discriminating power was statistically excellent between all treatment comparisons with the negative controls, 4) screening test results were reproducible across four tests, and 5 ) the screening test produced the same rank order for dentifrices as clinical studies that measured plaque reduction. In addition, 454 pyrosequencing of the PBBs indicated an oral microbial consortium was present. The most prevalent genera were Neisseria, Rothia, Streptococcus, Porphyromonas, Prevotella, Actinomyces, Fusobacterium, Veillonella and Haemophilus. We conclude these in vitro methods offer an efficient, effective and relevant screening tool for reduction of intact biofilm activity by dentifrices. Moreover, dentifrice rankings by the in vitro test method are expected to predict clinical results for plaque reduction.
\end{abstract}

(c) 2015 Elsevier B.V. All rights reserved.

\section{Introduction}

Caries and gingivitis continue to be prevalent oral health issues globally for children and adults in developed and developing countries (WHO, 2012, 2014). The best means to prevent and control caries and gingivitis is generally recognized as removal and control of bacterial plaque by daily brushing (Loe, 2000). Demonstrating new dentifrices have improved plaque reduction requires clinical studies. Predicting which new dentifrice formulations should progress to clinical studies has become problematic because in vitro methods to evaluate oral biofilm cleaning efficacy have not kept pace with dentifrice improvements.

The challenge was to find a simple rapid method to culture adequate amounts of oral biofilm that resisted cleaning and could be used for weekly high throughput screening. The biofilms would have to be representative of dental plaque which resists complete physical and chemical cleaning during routine oral hygiene. We reasoned that, just as routine brushing of teeth selects for residual plaque that must be

\footnotetext{
* Corresponding authors.

E-mail addresses: brucetepper@yahoo.com (B. Tepper), howard.bw@pg.com (B. Howard)

${ }^{1}$ Retired.
}

removed by dental prophylaxis, high shear during biofilm culturing would be needed to select for similar biofilm with a combination of high adhesion to physical substrate and high cohesion within the biofilm. Ideally a test method using such biofilms to discriminate cleaning efficacy of new dentifrice formulations would predict their clinical reduction of plaque.

Existing in vitro biofilm culturing and testing models did not meet our needs (see reviews: Sissons, 1997; Coenye and Nelis, 2010; Lebeaux et al., 2013; Salli and Ouwehand, 2015). Batch microtiter plate biofilm models such as Calgary Biofilm Device and Biofilm Ring Test are simple and capable of high throughput screening at relatively low cost, but the biofilms do not resist realistic in vitro cleaning due to minimal shear force during culturing. Lower throughput flow displacement models that culture with some shear such as the Centers for Disease Control Reactor, Modified Robbins Device, flow cells of various types and sizes, and the Constant Depth Film Fermentor along with other rotating disc reactors produce biofilms with limited resistance to cleaning and are not amenable to high throughput testing. Benchscale chemostats modified to continuously culture PBBs under high shear have appeal. Like their industrial-scale counterparts they can be adapted to study kinetics, mass-transfer and biodegradation (Nicolella et al., 2000). Chemostats, however, are challenging to maintain, 
expensive to purchase and operate, and require expertise to produce consistent biofilms over time.

We chose to pursue a combination of PBBs, batch culturing and 96well plate testing. We reasoned that biofilms that resist cleaning can be created by culturing biofilms on particles with various levels of non-laminar or discontinuous mixing to create shear force via highfrequency particle collisions. Using batch systems requires the least effort and expense while offering flexibility of culturing conditions. Once produced, PBBs can be distributed into 96-well plates and quickly separated from liquids by settling for various procedures and high throughput testing. In addition, shear conditions via particle collisions can be created during a test such that chemical and/or physical cleaning can be evaluated. Moreover, multiple endpoints can be measured in the same test on the same treatment replicate because each replicate treatment well contains hundreds of PBBs that can be parsed across multiple assay plates. In addition, fundamental research involving kinetics and biofilm community responses to stress would be possible.

One key issue to resolve was which particles to use. Oral PBBs were used in the 1970s to evaluate anti-plaque agents (Sudo et al., 1976), effects of culture conditions on oral biofilm species composition (Sudo, 1977), and adhesion and aggregation of oral bacteria to saliva-coated and uncoated surfaces (Clark et al., 1978; Wheeler et al., 1979). These early PBBs were cultured on either glass beads or glass beads coated with hydroxyapatite to improve colonization. Bacterial cells were recovered from these biofilms for viable cell counts to compare treatment effects. This was possible primarily because these biofilms had only modest adhesion and cohesion. Our initial trials with different sizes of glass beads with high shear culturing conditions demonstrated the expected; biofilm formation was minimal. Moreover, any hydroxyapatite coating would likely shear off the glass. The solution was to use hydroxyapatite particles of an appropriate size range as a way to manage shear forces due to collisions.

The other key issue to resolve was an appropriate rapid and applicable measure of treatment effects. Biofilms with high adhesion to substrate and cohesion within the biofilm resist cleaning and, therefore, would not easily release bacterial cells for viable cell counts. In addition, a more rapid and less expensive endpoint than cell counts was desired. Adenosine triphosphate (ATP) was chosen as the primary metric for cleaning efficacy. In our new model treated PBBs are compared for reduction of intact biofilm activity after treatment. Less ATP associated with treated intact biofilm than a negative control represents a combination of cell death, inhibition and/or dispersion. To distinguish among these causal factors requires additional metrics.

After developing our PBB culturing methods and a manual dentifrice cleaning test we migrated the test method to a robotic fluid handler. This reports on a single-lab validation of the automated method to demonstrate test results are sufficiently precise to distinguish treatment effects over a wide dynamic range, robust to potential interferences, repeatable over time and relevant to clinical plaque reduction by dentifrices.

\section{Materials \& methods}

\subsection{Saliva}

For each test, first morning stimulated human saliva (prior to oral hygiene) was self-collected daily by five to seven donors for three days. Donors were 21 to 60 years old in good health with no prophylaxis or treatment for any oral disease within 30 days. Other donor criteria for at least $48 \mathrm{~h}$ prior to and during collection included: no use of mouthrinse, floss, toothpicks or dentifrice other than Crest Cavity Protection; no fever >38 C, communicable disease or oral infection; no use of tobacco products, oral antibiotics or steroids; no use of antihistamines, decongestants or other cold/flu/allergy medicines; and females could not be pregnant or lactating. All donors were required to brush their teeth unsupervised twice daily during a minimum two-day washout period and during the collection period using supplied Crest Cavity Protection dentifrice containing $0.243 \%$ sodium fluoride and a standard manual toothbrush. Donors chewed on supplied sterile pieces of paraffin with beeswax or polypropylene tubing each morning to collect 25 to $30 \mathrm{~mL}$ saliva in $100 \mathrm{~mL}$ sterile wide-mouth screw-cap containers. After collection the containers were sealed and chilled for transport to the lab. Daily for three days equal volumes of saliva, 20 to $25 \mathrm{~mL}$, from each donor were pooled in a sterile $500 \mathrm{~mL}$ Erlenmeyer flask with 10 to 12 sterile borosilicate glass beads, 5 to $6 \mathrm{~mm}$ diameter (CG-1101-04, Chemglass Life Sciences, Vineland, NJ, USA). Pooled saliva was sheared by vortex at ca. $100 \mathrm{rpm}$ for 60 to $120 \mathrm{~s}$. Sheared pooled saliva sans settled solids was diluted $50 \%$ with sterile $0.9 \%$ saline (NDC0338-0048-04, Baxter Healthcare Corp., Deerfield, IL, USA) to form sheared pooled saliva diluted (SPSD). SPSD was amended only on the first culturing day to ca. $1 \%$ sucrose using a filter-sterilized $10 \%$ sucrose stock (OmniPur® Sucrose, EMD Chemicals Inc., Gibbstown, NJ, USA).

\subsection{Biofilms}

For each test PBBs were cultured in eight $50 \mathrm{~mL}$ centrifuge tubes, each tube containing 725 to $775 \mathrm{mg}$ UV-sterilized hydroxyapatite powder (HAP), 53 to $124 \mu \mathrm{m}$ mean diameter (Clarkson Chromatography Products Inc., South Williamsport, PA, USA). Culture tubes containing HAP plus $20 \mathrm{~mL}$ SPSD with sucrose were sealed with caps, transferred to a 31 to $33 \mathrm{C}$ aerobic incubator, laid on their sides on a rocking platform (Model 200, VWR Scientific Products, Radnor, PA, USA) and held in place with $15 \mathrm{~cm}$ bungee cords. Rocking speed was set between 7 and 8 to keep particles continually moving and colliding. After ca. 22 and $46 \mathrm{~h}$ culture tubes were removed from incubation and stood upright for at least $1 \mathrm{~min}$ to settle PBBs to tube bottoms. From each tube 18.5 to $19 \mathrm{~mL}$ supernatant was aspirated and replaced with $20 \mathrm{~mL}$ fresh SPSD before continuing incubation. After 68 to $72 \mathrm{~h}$ culture tubes were removed from incubation and supernatant was again removed. PBBs were rinsed twice, each time with $20 \mathrm{~mL}$ sterile saline, including inverting tubes ca. 10 times to effect thorough mixing. The bulk of PBBs settled to tube bottoms after 60 s leaving only the smallest particles suspended to cloud rinse supernatants. Suspended PBBs and planktonic bacteria were removed in rinse supernatants and discarded. Little to no cloudiness was observed in $10 \mathrm{~mL}$ sterile saline added to each tube to enable transfer of PBBs into a reservoir. Note that when the test method (2.4) is performed manually PBBs are transferred into a $50 \mathrm{~mL}$ disposable pipetting reservoir. When the method is performed on an epMotion 5075 automated pipetting system (Eppendorf, Hamburg, Germany) PBBs are transferred into a $100 \mathrm{~mL}$ epMotion reservoir.

\subsection{Treatments}

Sterile $0.9 \%$ saline was the negative control and used to prepare all treatments. Sodium lauryl sulfate (Stepanol, Stepan Co., Winder, GA, USA) at $0.1 \%$ in saline was the positive control, which approximated SLS present in 1:10 diluted dentifrice treatments. Crest ProHealth Clinical Gum Protection, Crest ProHealth, Crest Cavity Protection and Colgate Total Clean Mint dentifrices were purchased from a supermarket in Mason, OH, USA. Dentifrice slurries were prepared within 30 min of use by diluting ca. $2 \mathrm{~g}$ dentifrice with saline $10 \times$ (weight to volume) and homogenizing for 10 to $12 \mathrm{~s}$ using an alcohol-sterilized immersible BioHomogenizer (Model M133/1281-0, Biospec Products, Inc., Bartlesville, OK, USA). Supernatant (14 mL) of each diluted dentifrice treatment was transferred into its own well in a 12-deepwell reservoir (82007294, VWR). Treatments were assigned the same plate columns in 96well plates for each test. Since plate location bias can occur in 96-well plate assays, replicated treatments were used to check for bias across the plate, as follows: Saline \#1 and \#2 were in columns 1 and 12, respectively; SLS \#1 and \#2 in 2 and 11; Cavity Protection \#1 and \#2 in 3 and 10; and Colgate Total \#1 and \#2 in 6 and 9. Plate bias would be indicated 
if there were significant differences between multiple replicated treatments in the same direction; e.g., if there were significant differences between Saline \#1 and \#2 and between SLS \#1 and \#2 in the same direction. Crest ProHealth and Crest ProHealth Clinical Gum were assigned to columns 4 and 5, respectively, near the center of the plate. (Results are not included for two dentifrices tested in columns 7 and 8 that are not marketed in North America and for which no common clinical results were available for comparison.)

\subsection{Test method}

Validation tests were performed on an Eppendorf epMotion 5075 robot. Robot programs were created to replicate the manual test method for increased throughput. Transfers of $400 \mu \mathrm{L}$ saline and four random $50 \mu \mathrm{L}$ aliquots of rinsed PBBs were made into all wells of a sterile 96-deepwell ‘dosing plate' (Product 502.302, Eppendorf). Dosing consisted of rapidly dispensing $1 \mathrm{~mL}$ treatments into one column of dose plate wells followed by an immediate vigorous $1 \mathrm{~mL}$ aspiratedispense mix. After ca. $15 \mathrm{~s}$ during which PBBs settled to well bottoms $1 \mathrm{~mL}$ supernatant was slowly aspirated from dosed wells and discarded. One milliliter Dey-Engley Neutralizing Broth (Himedia, VWR) was rapidly dispensed with vigorous mixing 60 to $65 \mathrm{~s}$ after the dose was dispensed to arrest any further antimicrobial activity. The time between initial dosing and neutralization was chosen to approximate consumers' common brushing time with dentifrice. After ca. $15 \mathrm{~s}$ PBB settling $1 \mathrm{~mL}$ neutralizer supernatant was aspirated and discarded. Each column of wells was dosed and neutralized in succession. PBBs were then rinsed with mixing by column twice with $1 \mathrm{~mL}$ saline to dilute both residual treatment and neutralizer, either of which might affect the ATP endpoint (see 2.6). Pipetting tips were changed frequently to avoid crosswell contamination. Two random $50 \mu \mathrm{L}$ aliquots of dosed, neutralized and rinsed PBBs were transferred from each dosing plate well into the respective wells of three sterile 96-well assay plates, enabling up to three independent endpoints. PBBs accounted for ca. $30 \%$ of the $100 \mu \mathrm{L}$ transferred to assay wells. The first assay plate for the ATP analyses was black (Costar 3916, Corning Inc., Corning, NY, USA).

\subsection{Endpoint}

BacTiter-Glo ${ }^{\mathrm{TM}}$ (BTG) Microbial Cell Viability Assay (Promega Corp., Madison, WI, USA) was chosen for its sensitivity, dynamic range, reproducibility and our positive experience with the reagents. To each well containing dosed, neutralized and rinsed PBB sample was added $92 \mu \mathrm{L}$ BTG. Assay plates were incubated in the dark at 21 to $23 \mathrm{C}$ (ambient) with orbital mixing at $750 \mathrm{rpm}$ for $10 \mathrm{~min}$ on a Titramax 1000 (Heidolph Instruments $\mathrm{GmbH} \& \mathrm{Co}$., Schwalbach, Germany). Total incubation time from addition of BTG to last plate column wells to first well reading was 10.5 to $11.0 \mathrm{~min}$. Intracellular ATP released into the aqueous phase was used in the luciferase reaction. Emitted light was measured top down for $1 \mathrm{~s}$ per well as Relative Luminescence Units (RLU) on a Victor X5 2030 plate reader (Perkin Elmer, Waltham, MA, USA). [A temporary plate reader malfunction during test 4 resulted in a 16 min incubation. The additional incubation time was estimated to reduce potential RLU signal from wells by 4.5 to $14 \%$ based on additional readings of the same assay plate after $25 \mathrm{~min}$. The absolute differences in RLUs for study 4 did not change treatment rankings and no adjustments to data were made to account for this error.]

\subsubsection{Data transformations}

Untransformed RLU data were used to determine dynamic range, linearity and precision of the bioluminescence response. Log10transformed RLUs were used for treatment comparisons in figures and analyses.

\subsection{Linearity and reagent interference}

Eight materials were used to evaluate linearity and potential worstcase interference on the ATP response over a 1000-fold range. The materials were $0.9 \%$ saline, $0.05 \%$ cetyl pyridinium chloride (CPC), $0.1 \%$ SLS, Listerine Cool Mint mouthrinse, Dey-Engley neutralizer broth and 1:10 dilutions in $0.9 \%$ saline of three tested dentifrices-Cavity Protection, ProHealth and Colgate Total. Abiotic simulated supernatant types were created to contain approximate proportions of dosing solution, neutralizing broth and saline expected to be in supernatant samples from dosing, neutralizing and both rinsing steps of the test method. The 32 supernatant types were measured into replicate wells of assay plates at the same $100 \mu \mathrm{L}$ volume used for PBB samples. Four ATP standards $-1.0,0.1,0.01$ or $0.001 \mu \mathrm{M}$-were added to three replicate wells for each supernatant type. ATP activity was determined with BTG as above. Linearity of ATP response was determined by linear regression of RLUs on ATP standards within supernatant type for each test material. Absence of significant interference with the ATP response was determined for each ATP level by nested ANOVA of test material within supernatant type using all means comparisons by Tukey HSD (Tukey, 1949). Interference existed if the mean of log10 RLU readings for any material was significantly $(\alpha=0.05)$ depressed or amplified when compared to saline control within each supernatant type. The intact biofilm activity endpoint was considered robust if all second rinse supernatants were equivalent to saline control. Results for six of the 96 simulated dose supernatant wells were excluded from these analyses due to incorrect well volumes.

\subsection{Dynamic range and discriminating power}

The method of Zhang et al. (1999) for dynamic range was modified (Eq. (1)) to use mean response of the negative control, a high bioluminescence value, and minimum acceptable value (MAV) instead of mean response of the positive control. MAV was substituted because some test treatments had a lower ATP response than the $0.1 \%$ SLS positive control. MAV was conservatively set as $300 \mathrm{RLU}$, ca. 10 times background.

Dynamic range of method $=\left|\mu_{c-}-M A V\right|$,

where:

$\mathrm{u}_{\mathrm{c}-} \quad$ mean of negative (saline) control response and MAV minimum acceptable value.

Z-factors (Zhang et al., 1999) (Eq. (2)) estimate discriminating power using dynamic ranges and variation of responses. Replicated treatments were compared individually to each saline control within each test.

$Z_{t}=1-\left(\left(3 \sigma_{c-}+3 \sigma_{t}\right) /\left|\left(u_{c-}-u_{t}\right)\right|\right)$,

where:

$u_{t} \quad$ mean of any treatment response,

$\sigma_{c-} \quad$ standard deviation of negative control response, and

$\sigma_{t} \quad$ standard deviation of a treatment response.

\subsection{Repeatability}

Repeatability was determined as equivalent treatment rankings across replicate tests. One-way ANOVAs were performed on logtransformed intact biofilm activity for each of the four replicate tests. 
Treatment rankings were determined by all means comparisons using Tukey HSD $(\alpha=0.05)$.

\subsection{Verification}

Rankings of dentifrices based on intact biofilm activity reduction from the in vitro test were compared to rankings of the same dentifrices based on plaque reduction from clinical studies.

\subsection{Species composition}

During the first PBB test on each culturing day a $1 \mathrm{~mL}$ sample of diluted pooled saliva (2.1) in a $2 \mathrm{~mL}$ Eppendorf tube was centrifuged (Model 5415D, Eppendorf, Hauppauge, NY) at 14,000 rpm for $3 \mathrm{~min}$ to form a bacterial pellet. Supernatant was aspirated and discarded. In addition, three $200 \mu \mathrm{L}$ samples of the saline washed PBBs (2.2) to be used in the PBB test (2.4) were settled in $2 \mathrm{~mL}$ Eppendorf tubes, ca. $100 \mu \mathrm{L}$ excess supernatant was aspirated and discarded. The bacterial pellets and condensed PBB samples were stored at -15 to $-20 \mathrm{C}$ until thawed in an ice bath for DNA extraction. DNA was extracted using the Qiagen DNeasy Blood and Tissue Kit (Qiagen, Inc., Valencia, CA, USA) following the protocol "Pretreatment for Gram-Positive Bacteria” (In: DNeasy ${ }^{\circledR}$ Blood \& Tissue Handbook, 2006) with the following modifications. After resuspending samples in $180 \mu \mathrm{L}$ lysozyme solution $(20 \mathrm{mg} / \mathrm{mL})$ and vigorously vortexed to uniformity, $6 \mu \mathrm{L}$ mutanolysin $(25,000 \mathrm{U} / \mathrm{mL})$ and $3 \mu \mathrm{L}$ lysostaphin $(4000 \mathrm{U} / \mathrm{mL})$ were added, the mixture was vortexed again, and samples were sonicated at $37 \mathrm{C}$ for $30 \mathrm{~min}$. After sonication and prior to adding Proteinase $\mathrm{K}$ and AL buffer each sample received $4 \mu \mathrm{L}$ Rnase $(100 \mathrm{mg} / \mathrm{mL})$ followed by ca. $15 \mathrm{~s}$ vortexing and $10 \mathrm{~min}$ incubation at 21 to $23 \mathrm{C}$ (ambient).
Spin columns received an extra 2 min centrifugation at 14,000 rpm in clean collection tubes immediately prior to their transfer to sterile $1.5 \mathrm{~mL}$ LoBind tubes. Elution buffer AE was reduced from 200 to $100 \mu \mathrm{L}$ and the standing elution incubation was increased from 1 to $5 \mathrm{~min}$. Each $100 \mu \mathrm{L}$ extracted DNA sample was measured for quantity and quality by Nano Drop (Nano Drop ND-2000 Spectrophotometer, Thermo Scientific, Wilmington, DE, USA). The DNA samples were divided in half and frozen -15 to $-20 \mathrm{C}$. One set of the saliva and PBB DNA samples were express shipped to the Chinese Academy of Sciences for 454 pyrosequencing using the methods of Huang et al. (2011). Pyrosequencing of the barcoded 16S rRNA amplicons for the V1-V3 hypervariable regions was performed on a Genome Sequencer FLX Titanium (Roche, USA).

Statistical analyses were performed and figures prepared with JMP version 11.1.1 (SAS Institute, Cary, NC, USA).

\section{Results}

\subsection{Linearity and reagent interference in dosing and rinse \#2 supernatants}

ATP assay results (Fig. 1a and b) within all treatments within all supernatant types were linear $\left(\mathrm{R}^{2}>0.98\right)$ across a 1000 -fold range of ATP. Linearity was irrespective of observed interference. Simulated dosing supernatants of Cavity Protection and ProHealth dentifrices, and of Dey-Engley broth, significantly depressed ATP signal (Fig. 1a). Interference was absent in rinse \#2 supernatants (Fig. 1b) except for a nonsignificant $<0.1 \log$ mean signal deviations for ProHealth. These results confirmed preliminary findings that treatment material and neutralizer interference was minimized by 20 -fold dilution achieved by two saline rinses.

(a) Simulated treatment dosing supernatants

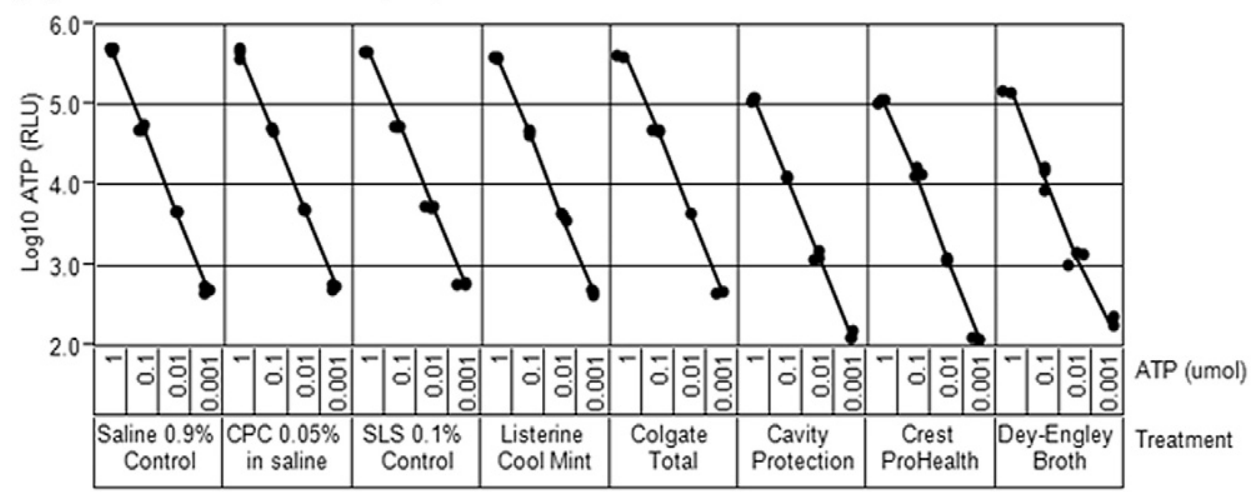

(b) Simulated saline rinse \#2 supernatants

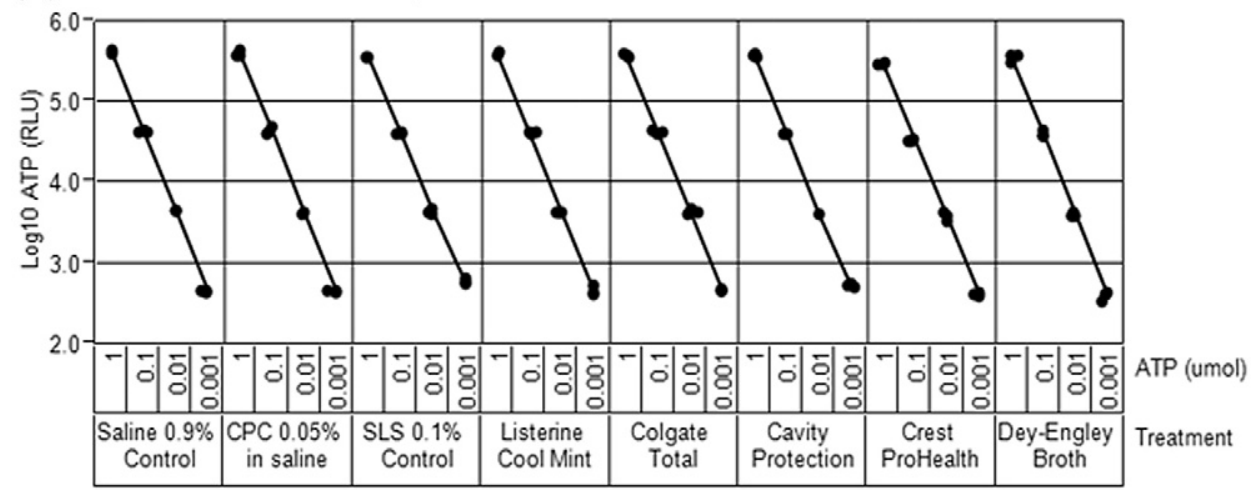

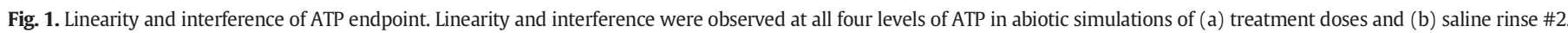
Treatment doses of Cavity Protection, Crest ProHealth and Dey-Engley Broth produced interference. Dilution achieved by two saline rinses eliminated the interference. 


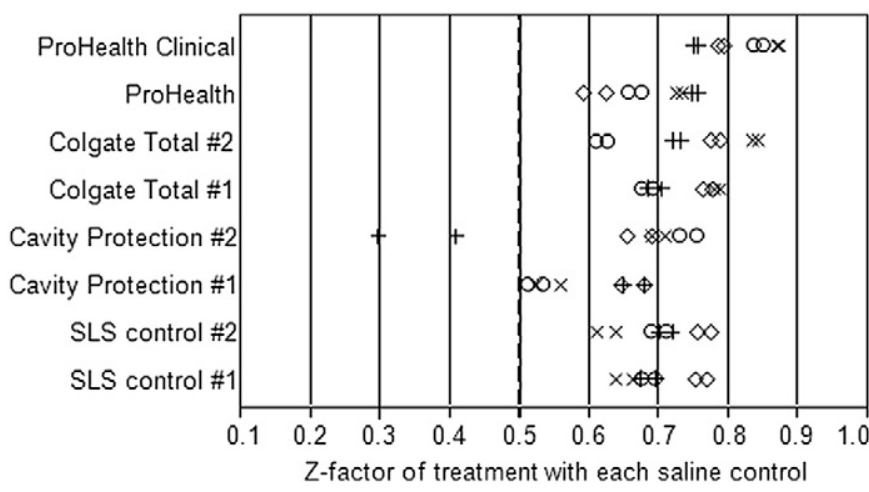

Fig. 2. Test method discriminating power. Z-factors were calculated within each test for all possible paired comparisons between each treatment and each saline control; $(O)$ Test 1 , (x) Test 2, $(\diamond)$ Test 3, and $(+)$ Test 4. Z-factors are a function of dynamic range and precision for a given screening test, where $1.0>Z>0.5$ reflects a wide dynamic range and excellent precision and $0.5>Z>0.3$ indicates narrower dynamic range and moderate precision for a test method (Zhang et al., 1999). Each replicated numbered treatment had $\mathrm{N}=8$ replicates.

\subsection{Dynamic range}

Intact biofilm activity measured as ATP bioluminescence had a maximum response range $\geq 1000$-fold. This is based on the minimum acceptable value (MAV) set at 300 RLU and typical observed saline control responses $>300,000$ RLU per PBB assay well (Fig. 3).

\subsection{Discriminating power}

Across all four tests intact biofilm activity for 70 of 72 comparisons between treatments and their respective saline controls had excellent precision based on $\mathrm{Z}$ factors between 0.5 and 1.0 (Fig. 2).

\subsection{Repeatability and robustness to processing variation}

Relative rankings of test materials were consistent across four tests despite observed plate bias for some replicate treatments within study
(Fig. 3, Table 1). Replicated saline and replicated SLS treatments at either ends of the ATP assay plate differed significantly $(p<0.05)$ and in the same direction in studies 2 and 3 (Table $1 \mathrm{~b}$ and $\mathrm{c}$ ). This plate bias was absent in studies 1 and 4 (Table 1a and d). Despite occasional plate bias the saline controls always had significantly higher intact biofilm activity than the other treatments (Table 1a to d) and treatment rankings were consistent across tests. Overall, dentifrices ranked from most to least reduction of intact biofilm activity as follows: ProHealth Clinical Gum Protection, ProHealth, Colgate Total, Cavity Protection.

\subsection{Verification}

Dentifrice ranking by in vitro reduction of intact biofilm activity was the same as dentifrice ranking by clinical plaque reduction (Barker et al., 2011; He et al., 2009, 2011, 2012, 2013; Sharma et al., 2013). Results from the six clinical studies (Table 2) can be summarized from most to least plaque reduction as follows: ProHealth Clinical Gum Protection, ProHealth, Colgate Total, Cavity Protection.

\subsection{Species composition}

The ten most prevalent genera and their relative distributions differed between the three day old PBBs used in Test 1 and the pooled saliva used for culturing them (Fig. 4). Streptococcus, Prevotella, Actinomyces and Veillonella accounted for two thirds of genera found in the saliva. In contrast, Neisseria, Rothia and Streptococcus accounted for more than $75 \%$ of PBB genera with Neisseria alone accounting for $50 \%$. Other genera found in PBBs in decreasing order were Porphyromonas, Prevotella, Actinomyces, Fusobacterium and Veillonella. Leptotrichia and Gamella were among the 10 most prevalent genera in saliva, but not in PBBs. Porphyromonas and Fusobacterium were among the 10 most prevalent genera in PBBs, but not in saliva.

\section{Discussion}

The Particle Based Biofilm culturing and test methods were developed for rapid inexpensive screening of biofilm cleaning efficacy by dentifrices. Test method validation demonstrated screening results were reproducible and likely to predict clinical plaque reduction over

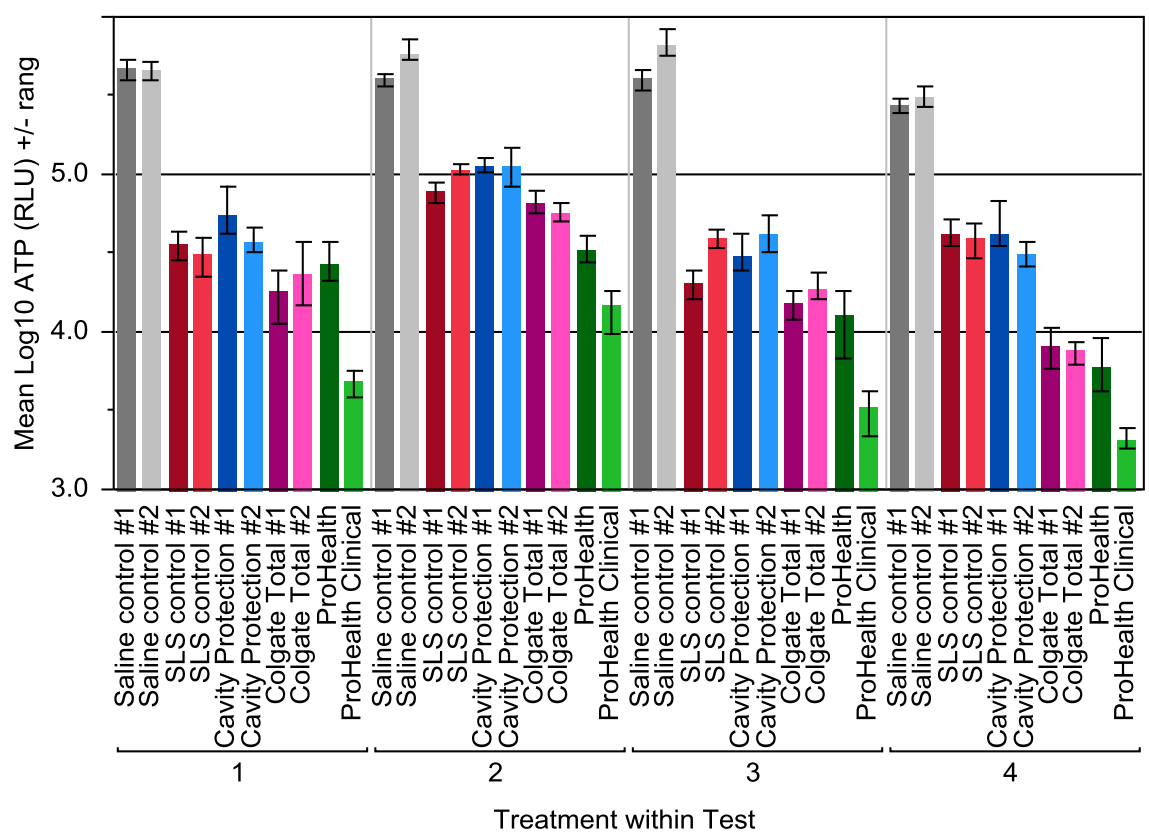

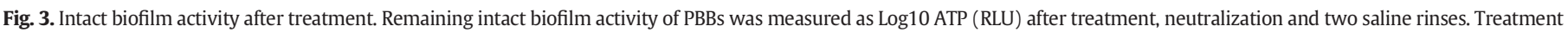
ranking were consistent among the four tests. 
Table 1

All treatment pair comparisons of intact biofilm ATP activity by Tukey HSD $(\alpha=0.05)$.

\begin{tabular}{|c|c|c|c|c|c|c|c|}
\hline \multicolumn{8}{|c|}{ (a) Study $1\left(R^{2}=0.98\right)$} \\
\hline Treatment & & & & & & & LS Mean \\
\hline Saline control 1 & A & & & & & & 5.673 \\
\hline Saline control 2 & A & & & & & & 5.658 \\
\hline Cavity Protection 1 & & B & & & & & 4.739 \\
\hline Cavity Protection 2 & & & C & & & & 4.572 \\
\hline SLS control 1 & & & C & $\mathrm{D}$ & & & 4.558 \\
\hline SLS control 2 & & & C & $\mathrm{D}$ & $\mathrm{E}$ & & 4.488 \\
\hline ProHealth & & & & $\mathrm{D}$ & $\mathrm{E}$ & & 4.429 \\
\hline Colgate Total 2 & & & & & $\mathrm{E}$ & $\mathrm{F}$ & 4.368 \\
\hline Colgate Total 1 & & & & & & $\mathrm{~F}$ & 4.263 \\
\hline ProHealth Clinical & & & & & & G & 3.687 \\
\hline
\end{tabular}

(b) Study $2\left(R^{2}=0.99\right)$

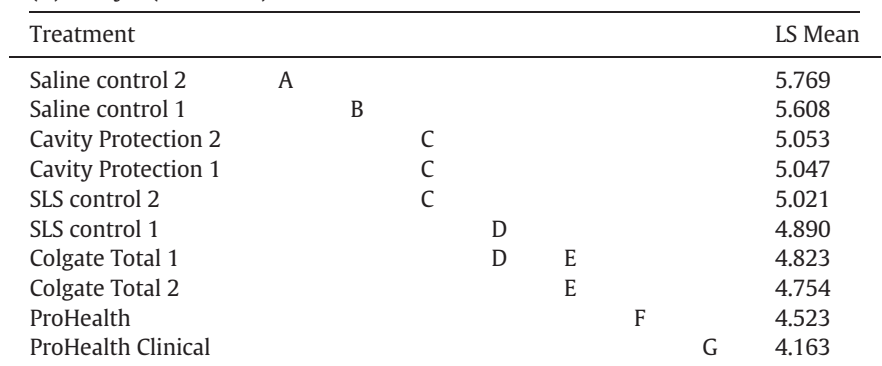

(c) Study $3\left(\mathrm{R}^{2}=0.99\right)$

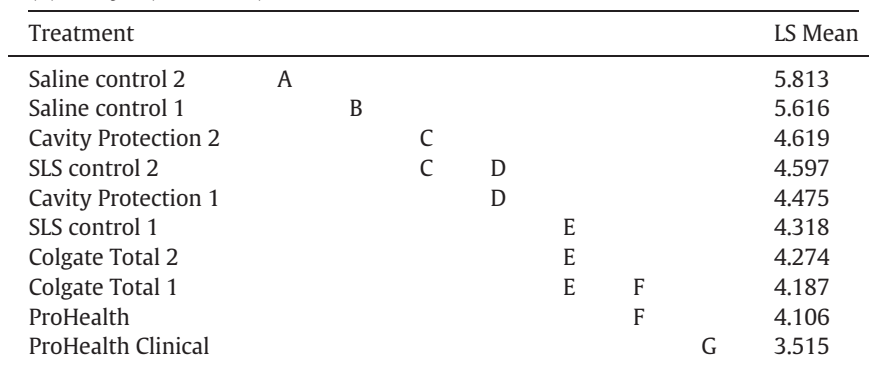

(d) Study $4\left(R^{2}=0.99\right)$

\begin{tabular}{|c|c|c|c|c|c|c|}
\hline Treatment & & & & & & LS Mean \\
\hline Saline control 2 & A & & & & & 5.494 \\
\hline Saline control 1 & A & & & & & 5.438 \\
\hline SLS control 1 & & B & & & & 4.629 \\
\hline Cavity Protection 1 & & B & & & & 4.622 \\
\hline SLS control 2 & & B & C & & & 4.601 \\
\hline Cavity Protection 2 & & & C & & & 4.493 \\
\hline Colgate Total 1 & & & & $\mathrm{D}$ & & 3.914 \\
\hline Colgate Total 2 & & & & D & $\mathrm{E}$ & 3.881 \\
\hline ProHealth & & & & & $\mathrm{E}$ & 3.777 \\
\hline ProHealth Clinical & & & & & $\mathrm{F}$ & 3.312 \\
\hline
\end{tabular}

Treatments with the same letter rank within each study are not significantly different.

a wide range of cleaning efficacy. In addition, the linear response and dynamic range of the endpoint will enable evaluation of future improvements in dentifrice formulations.

Table 2

Dentifrice rankings for plaque reduction in clinical studies.

\begin{tabular}{|c|c|c|c|c|c|c|c|}
\hline Ranking & & & & & & & References \\
\hline $\begin{array}{c}\text { ProHealth } \\
\text { Clinical }\end{array}$ & $>$ & ProHealth & $>$ & & & $\begin{array}{l}\text { Cavity } \\
\text { Protection }\end{array}$ & 1 \\
\hline \multirow[t]{3}{*}{$\begin{array}{c}\text { ProHealth } \\
\text { Clinical }\end{array}$} & $>$ & & & Colgate Total & $>$ & $\begin{array}{l}\text { Cavity } \\
\text { Protection }\end{array}$ & 4 \\
\hline & & ProHealth & $>$ & Colgate Total & & & $3,5,9$ \\
\hline & & ProHealth & $>$ & & & $\begin{array}{l}\text { Cavity } \\
\text { Protection }\end{array}$ & 6 \\
\hline
\end{tabular}

Note: '>' means dentifrice at left reduced plaque significantly $(\mathrm{p}<0.05)$ more than dentifrice at right.
Our simple batch culture system effectively selected for biofilms with high adhesion to physical substrate and high internal cohesion. Only biofilms on particles with these traits survived the continuous high-frequency collisions. Culturing biofilms on hydroxyapatite particles 53 to $124 \mu$ mean diameter proved critical to success. Particles needed to be large enough to support multi-species biofilm growth and small enough to be readily suspended for collisions and mixing during culturing and testing. Particles also needed to be large enough to settle quickly for separation from culturing medium and testing solutions yet small enough to be processed using standard multi-channel pipettors for high throughput testing. We learned during early method development that loss of particles that did not settle within $15 \mathrm{~s}$ were inconsequential to test results. These particles smaller than ca. $50 \mu$ did not support sufficient, if any, biofilm for testing. Particles larger than ca. $200 \mu$ were too heavy for high-frequency collisions during culturing and testing when using inexpensive and common laboratory equipment. More importantly, researchers and robotic fluid handlers were unable to consistently distribute larger particles evenly across dosing plate wells and subsequently to multiple assay plates.

While PBBs are amenable to 96-well plate testing, they introduce an additional level of bias. During test method development we observed that any pronounced bias in distribution of PBBs across dosing plate wells tended to be replicated in assay plates. This PBB distribution bias in assay plates increased variation in results which reduced the discriminating power between treatments. When the method was performed manually an analyst could correct for this plate bias by rejecting visually-unequal aspirated amounts of PBBs across the tips of a multichannel pipetor and resample PBBs for transfer. Immediate correction for this primary cause of plate bias is not possible when using robotics. Robot programs must minimize this sampling bias specifically for the robot being used and the programmer's implementation of the method. Repeated and careful observations led to the aspiration, mixing and dispensing rates used for PBB transfers that minimized plate bias in study 4 and proved to be satisfactory during three years of subsequent testing. What is remarkable is that results of method validation tests were robust to significant plate bias during two of the tests. Relative treatment rankings were the same across studies and discriminating power remained high. Despite the apparent method robustness to plate bias, careful robotic implementation of the method is recommended based on our implementations on two fluid handlers, an Eppendorf epMotion ${ }^{\circledR} 5075$ and more recently on a Biomek ${ }^{\circledR}$ NXp Span-8 (Beckman Coulter, Brea, CA, USA).

ATP proved a wise choice as an alternative endpoint to viable cell counts. The time and expense to consistently disperse cells without injury for viable cell counts from biofilm selected for high adhesion and cohesion was considered ill-advised and proved challenging during initial method development. In vivo samples of oral plaque, presumably with similar levels of biofilm adhesion and cohesion to tested PBBs, were reportedly difficult to disperse into single cells for accurate viable cell counts (Loesche et al., 1972). In addition, viable cell counts can misrepresent level of biofilm activity, particularly when many biofilm cells may be dormant due to nutrient limitations and/or treatment with inhibitory substances. In contrast, ATP is a precise and valid metric previously applied to similar oral biofilm testing. ATP of dispersed oral biofilm cells after treatment was successfully used to quantitate relative efficacy of mouthrinses and mouthrinse ingredients (Pan et al., 2010; Sánchez et al., 2013). Sánchez et al. (2013) also found that viable cell counts and ATP levels were significantly $(\mathrm{p}<0.001)$ correlated. Our experience indicates intact biofilm less than ca. $20 \mu$ thick can be directly measured reproducibly for ATP activity using BacTiter-Glo ${ }^{\mathrm{TM}}$ without needing to first disperse the biofilm.

Importantly, we interpret remaining intact biofilm to have reduced ATP levels when less biofilm is physically present on particles and/or some retained biofilm bacteria are dead or metabolically inhibited by treatments. Any of these situations result in slower regrowth which helps control biofilms. We make no attempt here to link ATP levels 


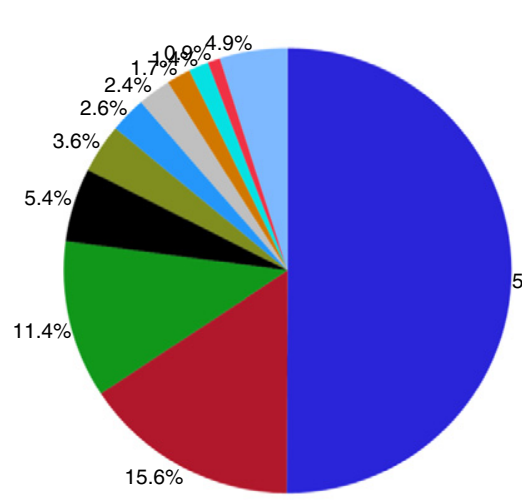

Particle Based Biofilms

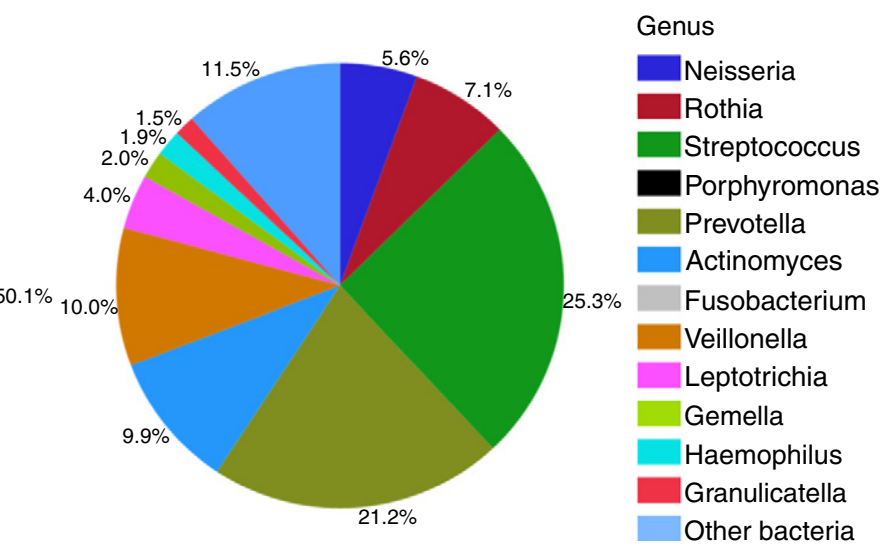

Pooled Saliva

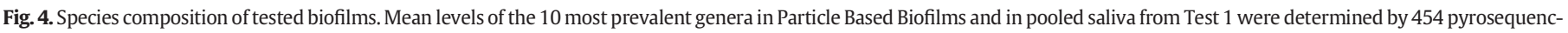

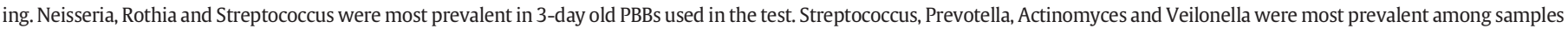
from each day's pooled saliva used to culture the PBBs.

with cell counts, nor do we link ATP activity of intact biofilm to a specific amount of biofilm on particles. Rather, for the cleaning test method to be valid, we stress that 1 ) all dose plate wells need to start with approximately equal amounts of PBBs with equivalent levels of total biofilm activity and 2) the distribution of treated PBBs to assay plates needs to be unbiased. These are the key parameters to check when performing the test method manually or when automating it with robotics.

Perhaps the most interesting findings involved the microbial consortia of three day old PBBs used in Test 1. Most of the predominant genera found on the PBBs, Neisseria, Rothia, Streptococcus, Actinomyces, Veillonella and Haemophilus, are pioneer species common in supragingival plaque and able to tolerate aerobic conditions (Aas et al., 2005; Marsh and Martin, 2009). The other most prevalent genera on PBBs, Porphyromonas, Prevotella and Fusobacterium, are known to attach subsequently (Marsh and Martin, 2009) suggesting the expected succession of colonizing species took place to some extent under the high shear culturing conditions used. The relative distribution of genera on the PBBs was markedly different than found in the pooled saliva used in culturing. The obvious implication is that growth of some species occurs principally in biofilms and is somewhat independent of the levels of dispersing genera found in saliva. For example, Porphyromonas and Fusobacterium were among the most prevalent genera on PBBs despite their very low levels in the saliva. In addition, the range of genera found on PBBs suggests that a microbial consortia had formed in three days using simple daily renewal of diluted saliva. Studies are progressing to understand development of PBB consortia under varying culture conditions, including the observed predominance of Neisseria on young PBBs.

Why Neisseria were predominant on the PBBs is unclear. Benign species of Neisseria are ubiquitous in the human oral microbiome and can be found at relatively low levels on both hard and soft tissue (Liljemark and Gibbons, 1971; Aas et al., 2005; Marsh and Martin, 2009). Analysis of in vivo samples indicated Neisseria colonization on newly cleaned teeth and on established plaque in ten subjects was low, representing $<2 \%$ of cultivable species present in both situations (Liljemark and Gibbons, 1971). We suspect that the high shear culturing conditions we used and/or differences between tooth enamel (in vivo) and hydroxyapatite particles (in vitro) selects for high early colonization by Neisseria. Studies are progressing to determine the causal factors.

In addition to the above on-going studies, the PBB model can be used to investigate diverse and complex hypotheses because of the ease and flexibility of the culturing methods, the 96-well plate venue and the ability to measure multiple endpoints on the same replicated treatment wells. We have explored how modifying culture conditions can artificially select for caries- and gingivitis-like biofilm communities. Early studies indicate monitoring community shifts in vitro may be a viable additional screening endpoint for mouthrinse and dentifrice prototypes. In addition, complex multi-level study designs are possible in individual tests. For example, PBBs from different culturing regimes can be exposed to a suite of treatments for different exposure durations and replicates can be measured for intact biofilm activity and up to two additional endpoints.

Finally, inter-operator and inter-laboratory reproducibility was demonstrated within Procter \& Gamble labs after validation studies were completed. Three contractors and three P\&G researchers all successfully cultured and performed PBB studies. Rigorous blinded interlaboratory reproducibility studies at other labs remain to be performed.

\section{Conclusions}

PBB culturing and test methods have been demonstrated to be an efficient, precise and reproducible way to evaluate cleaning efficacy of dentifrices. The potential for this screening method to predict clinical efficacy of future dentifrice prototypes for plaque reduction is promising based on equivalent rankings of marketed dentifrices.

\section{Acknowledgments}

We are grateful for an anonymous reviewer's comments that helped improve the manuscript. We thank Matthew Barker and Tao He for their help with identifying appropriate clinical studies for comparison with the in vitro test results. Rui Li coordinated work with the Chinese Academy of Sciences and made many helpful suggestions to improve the manuscript. This work was designed and funded by The Procter \& Gamble Company. All research was conducted at Procter \& Gamble, Mason, $\mathrm{OH}$, USA except for the 454 pyrosequencing conducted at the Chinese Academy of Sciences, Shandong, China.

\section{References}

Aas, J.A., Paster, B.J., Stokes, L.N., Olsen, I., Dewhirst, F.E., 2005. Defining the normal bacterial flora of the oral cavity. J. Clin. Microbiol. 43, 5721-5732.

Barker, M.L., Cheng, R., Dunavent, J.M., Biesbrock, A.R., He, T., 2011. Meta-analysis of clinical anti-plaque effectiveness for $0.454 \%$ stannous fluoride dentifrices. Abstract and PosterIADR 89th General Session.

Clark, W.B., Bammann, L., Gibbons, R.J., 1978. Comparative estimates of bacterial affinities and adsorption sites on hydroxyapatite surfaces. Infect. Immun. 19, 846-853.

Coenye, T., Nelis, H.J., 2010. In vitro and in vivo model systems to study microbial biofilm formation. J. Microbiol. Methods 83, 89-105.

He, T., Cheng, R., Baker, R., Biesbrock, A., 2009. Anti-plaque efficacy of $0.454 \%$ stannous fluoride dentifrices. Abstract and PosterIADR 87th General Session.

He, T., Barker, M., Sagel, P.A., Biesbrock, A.F., 2011. Anti-plaque efficacy of a $0.454 \%$ stannous fluoride dentifrice. Abstract and PosterIADR 89th General Session. 
He, T., Biesbrock, A., Barker, M.L., Eynon, H., Milleman, J., Milleman, K., Putt, M.S. Wintergerst, A.M., 2012. Anti-Plaque Benefits of Two Therapeutic Dentifrices Using DPIA.

He, T., Barker, M.L., Biesbrock, A.R., Eynon, H., Milleman, J.L., Milleman, K.R., Putt, M.S., Wintergerst, A.M., 2013. Digital plaque imaging evaluation of a stabilized stannous fluoride dentifrice compared with a triclosan/copolymer dentifrice. Am. J. Dent. 26 (6), 303-306.

Huang, S., Yang, F., Zeng, X., Chen, J., Li, R., Wen, T., Li, C., Wei, W., Liu, J., Chen, L., Davis, C. $\mathrm{Xu}, \mathrm{J} ., 2$ 2011. Preliminary characterization of the oral microbiota of Chinese adults with and without gingivitis. BMC Oral Health 11 (33), 1-14.

Lebeaux, D., Chauhan, A., Rendueles, O., Beloin, C., 2013. From in vitro to in vivo models of bacterial biofilm-related infections. Pathogens 2, 288-356.

Liljemark, W.F., Gibbons, R.J., 1971. Ability of Veillonella and Neisseria species to attach to oral surfaces and their proportions present indigenously. Infect. Immun. 4, 264-268.

Loe, H., 2000. Oral hygiene in the prevention of caries and periodontal disease. Int. Dent. J. $50,129-139$

Loesche, W.J., Hockett, R.N., Syed, S.A., 1972. The predominant cultivable flora of tooth surface plaque removed from institutionalized subjects. Arch. Oral Biol. 17, 1311-1325.

Marsh, P.D., Martin, M.V., 2009. Oral Microbiology. Fifth edition. Elsevier, London, pp. 30-59 (80-85).

Nicolella, C., van Loosdrecht, M.C.M., Heijnen, S.J., 2000. Particle-based biofilm reactor technology. Trends Biotechnol. 18, 312-320.

Pan, P.C., Harpera, S., Ricci-Nittela, D., Luxb, R., Shib, W., 2010. In-vitro evidence for efficacy of antimicrobial mouthrinses. J. Dent. 38 (Suppl. 1), S16-S20.

Salli, K.M., Ouwehand, A.C., 2015. The use of in vitro model systems to study dental biofilms associated with caries: a short review. J. Oral Microbiol. 7, 26149.
Sánchez, M.-C., Llama-Palacios, A., Marín, M.-J., Figuero, E., León, R., Blanc, V., Herrera, D., Sanz, M., 2013. Validation of ATP bioluminescence as a tool to assess antimicrobial effects of mouthrinses in an in vitro subgingival-biofilm model. Med. Oral Patol. Oral Cir. Bucal 18, e86-e92.

Sharma, M., He, T., Barker, M.I., Biesbrock, A.R., 2013. Plaque control evaluation of a stabilized stannous fluoride dentifrice compared to a triclosan dentifrice in a six-week trial. J. Clin. Dent. 24, 31-36.

Sissons, C.H., 1997. Artificial dental plaque biofilm model systems. Adv. Dent. Res. 11, $110-126$.

Sudo, S.Z., 1977. Continuous culture of mixed oral flora on hydroxyapatite-coated glass beads. Appl. Environ. Microbiol. 33, 450-458.

Sudo, S.Z., Schotzko, N.K., Folke, L.E.A., 1976. Use of hydroxyapatite-coated glass beads for preclinical testing of potential antiplaque agents. Appl. Environ. Microbiol. 32 (3), $428-432$.

Tukey, J.W., 1949. Comparing individual means in the analysis of variance. Biometrics 5 , 99-114.

Wheeler, T.T., Clark, W.B., Birdsell, D.C., 1979. Adherence of Actinomyces viscosus T14V and T14AV to hydroxyapatite surfaces in vitro and human teeth in vivo. Infect. Immun. $25,1066-1074$.

World Health Organization, 2012. Oral health fact sheet No. 318. http://www.who.int/ mediacentre/factsheets/fs318/en/index.html.

World Health Organization, 2014. What is the burden of oral disease? http://www.who int/oral_health/disease_burden/global/en/

Zhang, J.H., Chung, T.D.Y., Oldenburg, K.R., 1999. A simple statistical parameter for use in evaluation and validation of high throughput screening assays. J. Biomol. Screen. 4, 67-73. 